\title{
Cambiando prácticas en el campo de la ayuda internacional para el desarrollo: el ascenso de China y su influencia en África
}

\section{Changing Practices in the Field of International Development aid: The rise of China and its Influence on Africa}

DOI: $10.32870 /$ mycp.v10i30.745

\section{Resumen}

El presente trabajo tiene como objetivo analizar el impacto del surgimiento de China como donante no tradicional, enfocándonos en el caso particular de África. Debido a las peculiaridades que muestran las prácticas de ayuda chinas, el posicionamiento de Pekín como jugador importante en dicho campo representa un serio desafío al orden establecido, ejemplificado en las normas e ideas establecidas por el Comité de Ayuda para el Desarrollo (CAD) de la Organización para la Cooperación y el Desarrollo Económico (OCDE), que ha dominado desde el final de la Segunda Guerra Mundial. Así pues, para poder observar la tensión entre el surgimiento de China como donante y el orden imperante, el trabajo adopta un enfoque centrado en las prácticas sociales, que enfatiza las acciones como potenciadoras del cambio o como mantenedoras del statu quo, argumentando que para el caso chino en África se estarían posicionando dentro de la primera categoría.

Palabras clave: China, África, OCDE, ayuda internacional para el desarrollo, giro práctico.

\author{
Victor Manuel Olea Contreras ${ }^{1}$
}

\begin{abstract}
The present work seeks to analyze the impact of the emergence of China as a non-traditional donor, focusing on the African case. Due to the peculiarities of Chinese practices of aid, the arrival of the government of Beijing as a powerhouse within the field of international aid represents a challenge to the norms and ideas established by the Development Assistance Committee (DAC) of the Organization for Economic Co-operation and Development (OECD), which has dominated the field since the end of the Second World War. In that sense, to observe the tension between the emergence of China as a donor and the dominant order, the article adopts a practice approach, emphasizing the role of social practices as enhancers of change or as keepers of the status quo, arguing that in the case of China in Africa we are witnessing the first.
\end{abstract}

Keywords: China, Africa, OECD, international aid for development, practice turn.

Artículo recibido el 21 de enero de 2021 y dictaminado el 10 de marzo de 2021.

1. Instituto Tecnológico y de Estudios Superiores de Occidente (ITESO). Periférico Sur Manuel Gómez Morín 8585, San Pedro Tlaquepaque, Jalisco, México. ORCID: https://orcid.org/0000-0003-04933230. Correo electrónico: voleacontreras@gmail.com 


\section{Introducción}

Uno de los fenómenos que ha suscitado un intenso debate y ha encendido calurosas discusiones en materia de política internacional en los últimos años, es el del ascenso de China y las repercusiones que esto puede tener para el actual orden internacional. Desde la llegada de Deng Xiaoping como líder paradigmático del gigante asiático, tras la muerte de Mao Zedong en 1976, la República Popular de China entró en una trayectoria dependiente que lo ha convertido, de manera gradual y paulatina, en uno de los jugadores más importantes de la arena internacional. Si bien es cierto que hasta el día de hoy las implicaciones de dicho fenómeno todavía no se manifiestan de manera clara, es innegable que de una u otra manera el ascenso de China seguramente tendrá repercusiones debido a que representa un modelo económico distinto y un modelo de organización política diferente a aquel que impera a nivel mundial (Beeson, 2013, p. 242).

La investigación en torno al puzzle sobre el ascenso chino es amplia y los temas que abarca son extensos. Dentro de esos temas, uno que ha destacado particularmente, y del cual existe una vasta bibliografía, es el de la ayuda para el desarrollo. En ese sentido, parece que el surgimiento de China como "nuevo donante", categoría utilizada para nombrar a todos aquellos Estados que no se encuentran dentro del esquema del Comité de Ayuda para el Desarrollo (CAD) de la Organización para la Cooperación y el Desarrollo Económico (OCDE), ha logrado cimbrar el andamiaje institucional en torno a la entrega de ayuda y con esto está logrando modificar el paisaje internacional en dicho tema.

Así pues, y en sintonía con lo anterior, el objetivo del presente trabajo es analizar cómo China está cambiando el panorama de la ayuda para el desarrollo, y a través de qué mecanismos lo está haciendo. Dando respuesta a las interrogantes anteriores, en el estudio se plantea que China está modificando de manera progresiva la estructura normativa vigente en torno a la ayuda, proponiendo una visión basada en su propia experiencia de desarrollo y, además, enfatizando prácticas como la no interferencia en los asuntos domésticos de los países receptores, la construcción de grandes proyectos de infraestructura y resaltando el intercambio de dicha ayuda con la compra de bienes chinos o la extracción de recursos naturales o materias primas. Dichas prácticas, contrapuestas a aquellas que realizan los donantes tradicionales, se posicionan como una clara afrenta a la estructura establecida dentro de dicha esfera de la política internacional. 
Para poder comprobar lo anterior, se está tomando como caso la relación de China con el continente africano, ${ }^{2}$ debido al gran impacto que ha tenido el "Reino del Medio" en los últimos años en dicho continente. Después de Asia, zona de influencia natural para ejecutar sus políticas de ayuda, África se ha posicionado como la segunda región más importante para el economic statecraft del Gobierno de Pekín y, por ende, como una de las regiones en donde se entrega más ayuda. Además, la relación entre el continente y el país asiático resalta debido a que es objeto de gran escrutinio por parte de la comunidad internacional y de un gran número de especialistas en materia de cooperación, desarrollo y relaciones internacionales. Esto se debe, principalmente, a que la irrupción de China como donante en África parece estar modificando la arquitectura de la ayuda internacional que fue promovida por los países occidentales durante mucho tiempo. Esto ha provocado la suspicacia de un gran número de académicos, activistas y políticos, que ven la intromisión de China como una maldición para el desarrollo económico, político y social de gran parte de los países africanos. Otros, por su parte, tienen una visión más benévola sobre lo que puede brindar el nuevo enfoque, tomando como base la propia experiencia china.

Así pues, parece que sin tener una estructura normativa alterna estructurada claramente, ${ }^{4}$ el Gobierno de China está tratando de modificar, o por lo menos disputar, la arquitectura en torno a la ayuda que domina el tema. Derivado de esto, es necesario, entonces, buscar precisamente la forma cómo el gigante asiático está reconfigurando el campo de la ayuda internacional. Con ese fin, en el presente trabajo se aplica el marco teórico-conceptual del denominado giro práctico en relaciones internacionales, derivado del enfoque

2. Cabe notar que al hablar de la relación de China con el continente africano se está haciendo una sobre-simplificación, debido a la diversidad y complejidad que muestra la relación de Pekín con cada uno de los Estados que integran dicho continente, dejando de lado particularidades para cado caso específico. Sin embargo, para los objetivos que persigue el trabajo dicha conceptualización permite demostrar lo que se está argumentando.

3. Nombre con el cual se denomina, en algunos textos, a China. Dicha concepción proviene de la traducción de Zhongguo (中国), nombre como se le conoce a dicho país en el idioma chino.

4. Cabe hacer mención de que si bien algunos especialistas y académicos señalan al denominado "Consenso de Pekín" como la propuesta normativa del Gobierno chino para desplazar al denominado "Consenso de Washington", y a algunas otras normas internacionales, como la democracia o los derechos humanos, dicho "molde”, creado por el analista Joshua Copper Ramos, representa más una etiqueta impuesta al comportamiento pragmático, que en ocasiones muestra ser contradictorio del Gobierno chino (Givens, 2011). 
del sociólogo francés Pierre Bourdieu, para responder a las interrogantes que se plantean.

\section{El giro práctico de relaciones internacionales}

El enfoque de las prácticas en relaciones internacionales se desprende del trabajo de personajes como Emmanuel Adler, Vincent Pouliot, Rebecca AdlerNissen, Christian Bueger y Frank Gadinger, por mencionar solo algunos. Es necesario subrayar que dicho "giro", como se le ha denominado, no constituye un enfoque unificado, más bien se habla de un conjunto de perspectivas teóricas que ponen énfasis en el concepto de práctica social para entender la realidad internacional (Adler \& Pouliot, 2011, p. 4). Así pues, podemos encontrar estudiosos que toman como referencia el trabajo de Schelling (Adler \& Pouliot, 2011), de Bourdieu (Bigo, 2013; Adler-Nissen, 2011), de De Certeau (Neumann, 2002), de la escuela "pragmática" de James y Dewey (Kratochwil, 2011), entre otros. Derivado de esto, no podemos suponer que el giro práctico se encuentre constituido como una gran narrativa, sino como una serie de enfoques que tienen similitudes y diferencias, pero que convergen, precisamente, en el hecho de que ponen la práctica en el centro de atención (Cornut, 2017).

Dentro de todos los enfoques e influencias que existen en torno a las prácticas, y como bien lo mencionan Bueger y Gadinger (2015), a manera de crítica, el trabajo del sociólogo francés Pierre Bourdieu ocupa un lugar preponderante, asumiéndose, casi de manera automática, como la visión que más influencia tiene dentro de la disciplina de las relaciones internacionales. Si bien es cierto que la crítica que realizan los investigadores al enfoque bourdesiano es extremadamente aguda y válida, es necesario enfatizar que su aparato teórico-conceptual, por lo menos para analizar el fenómeno de la ayuda, es extremadamente útil y proporciona una visión poco explicitada dentro de los estudios especializados.

Para Bourdieu, y de manera general, una práctica social es una acción social, cualquier cosa que uno hace y que tiene significación social; una acción que se encuentra incrustada en una estructura compleja de significados objetivos e (ínter) subjetivos. Así pues, escuchar un disco de música clásica, ir a jugar golf, comer cierto tipo de alimentos, casarse, etc. pueden ser consideradas como prácticas sociales. Tomando como base dicha perspectiva micro-sociológica, se establece que esas acciones son fundamentales para entender cómo se 
conforman las estructuras de poder simbólico (Adler-Nissen, 2012, p. 5), aquello que crea legitimidad, creencia, obediencia y consenso entre quienes dominan y los dominados (Loyal \& Quilley, 2017). Precisamente para realizar la conexión entre una parte, la práctica, y la otra, las estructuras de poder simbólico, Bourdieu desarrolla los conceptos de campo, habitus y capital, los cuales a su vez permiten conocer las motivaciones y disposiciones de los individuos para realizar la acción social.

El campo es el espacio social, una estructura que, debido a la distribución de capital que existe entre las unidades que la componen, es jerárquica y asimétrica y, además, que surge de la lucha que se gesta entre éstas por apropiarse del recurso, capital o bienes que ofrece dicho campo (Cerón-Martínez, 2019; Chihu Amparán, 1996; Pouliot \& Mérand, 2013). Partiendo de la analogía de los campos gravitacionales, Bourdieu plantea que éstos funcionan con un centro, la idea sobre el cual se construye el campo, que atrae y permite mantener a las unidades que lo conforman dentro de su órbita (Aedo Henríquez, 2014, p. 59). En la realidad social existen un gran número de campos, los cuales son relativamente autónomos los unos de los otros, que varían en su tamaño y que en su conjunto constituyen la realidad social completa. Como bien especifica el autor, cada campo posee instituciones particulares y una lógica única que le permite funcionar y reproducirse (Bourdieu, 1987/2000, p. 108).

Dichos espacios cuentan con dos dimensiones que constituyen una pieza clave para entender las tensiones, o juegos, que se desarrollan dentro de ellos. La primera nos habla del condicionamiento que ejercen en las unidades que lo componen. En ese sentido, los campos fungen como estructuras objetivas que imponen condiciones a aquellos que se encuentran imbuidos en ellos. Alejándose de una posición idealista, Bourdieu sugiere que las posiciones jerárquicas que ocupan los integrantes de un determinado campo representan una estructura objetiva que existe más allá de la propia conciencia de los individuos o de sus deseos, que impone limitantes y modula las relaciones entre aquellos que se encuentran en posiciones más elevadas, dominantes, y los que se encuentran en posiciones más bajas, dominados (Pouliot, 2013, p. 47). Esto se logra por medio de lo que el propio teórico denomina como doxa, que son, a grandes rasgos, todas las ideas y creencias legitimadas y hechas verdades; las ideas incuestionables que imponen aquellos que se encuentran en una posición de mayor jerarquía (Bourdieu, 1994/1997, p. 121; CerónMartínez, 2019, p. 314) y que constituyen, de alguna manera, las pautas normativas que lo rigen. 
La segunda dimensión es la del conocimiento, la cual se encuentra conformada por los entendimientos que existen en torno al campo, el sentido del juego que le permite a las unidades saber cómo operar, cómo comportarse (Cerón-Martínez, 2019; Aedo Henríquez, 2014). Este sentido del juego conforma lo que Bourdieu denomina habitus, el cual se encuentra constituido por las experiencias históricas, personales y colectivas; normas sociales internalizadas; modos de pensar; prejuicios; creencias, etc. que adquieren los agentes a lo largo de su experiencia vital y que permiten crear principios que sirven de guías para poder dilucidar las acciones que deben de realizar (Cornut, 2017; Pouliot \& Mérand, 2013, p. 29). En ese sentido, el habitus bourdesiano es un elemento clave para entender las prácticas sociales, debido a que es el origen de éstas (Adler-Nissen, 2012). Como bien lo indica Chihu Amaparán (1996), las disposiciones o predisposiciones que conforman el habitus:

[...] no hacen referencia al carácter, moralidad o socialización, sino a propensiones clasificatorias y valorativas socialmente adquiridas y manifestadas a través de la apariencia externa, las opiniones y los fenómenos incorporados tales como la conducta, la postura, las maneras de caminar de sentarse, etcétera (pp. 185 y 186).

Tanto el concepto de campo como el de habitus constituyen las piezas clave para entender la propuesta teórica de Bourdieu. Esto se debe a que por medio de los dos intenta zanjar el problema agente/estructura que impera en las ciencias sociales. Así pues, a través de lo objetivo, el campo, y lo subjetivo, el habitus, el pensador francés construye un enfoque relacional que trata de superar las limitantes de los enfoques de acción racional y del enfoque sobresocializado del estructuralismo parsoniano (Pouliot \& Mérand, 2013, p. 31). De esta manera construye lo que se denomina "subjetividad socializada", aseverando que lo individual, lo subjetivo, es social y colectivo (Bourdieu \& Wacquant, 1992/2008, p. 186). Por tanto, puede pensarse en Bourdieu como un estructuralista, al asegurar que existen estructuras objetivas ajenas a los deseos de los individuos; y constructivista, al sugerir que existe "una génesis de los esquemas de percepción y de acción constitutivos de los habitus" (Chihu Amparán, 1996, p. 181).

Finalmente, y para cerrar esta breve presentación, tenemos la noción de capital. Para Bourdieu, el capital no puede ser reducido a una visión economicista de la realidad, como suponen algunos pensadores marxistas. En su visión, el capital tiene que ser entendido como un "recurso de cambio" que 
debe ser extendido a otros ámbitos de la realidad; una "moneda de cambio", en el sentido de que un tipo de capital permite intercambiarse por otro, que se traslada a los aspectos sociales (capital social y la movilización de recursos en función de la pertenencia a determinado grupo y organización social), de información (capital cultural, ejemplificado en los hábitos adquiridos durante el proceso de socialización), socioculturales y de orden simbólico (capital simbólico, ejemplificado en el poder de legitimar e imponer valores y estilos culturales, artísticos y morales) y económico (capital económico, constituido por recursos monetarios y financieros) (Cerón-Martínez, 2019, p. 313; Chihu Amparán, 1996, p. 184; Pouliot \& Mérand, 2013, p. 36). Además, la posesión de dicho capital, en todos sus sentidos es lo que permite conformar la estructura jerárquica de los diferentes campos que constituyen la realidad social. De acuerdo con el capital que se aprecie dentro del campo específico, su posesión permite que algunos agentes se encuentren en la posición de subordinantes y otros de subordinados (Chihu Amparán, 1996, pp. 183 y 184).

Así pues, y a manera de resumen, se puede pensar, como lo hace CerónMartínez (2019), en el enfoque de prácticas sociales de Bourdieu como una ecuación, en donde el origen de las prácticas sociales se gesta por medio de la suma del campo más la suma del habitus y capital, ejemplificado de la siguiente manera: campo + (habitus + capital $)=$ prácticas sociales .

\section{La creación del campo}

\section{El orden liberal y el régimen de ayuda}

Una vez que se ha trazado un esbozo general del "giro práctico" y se ha explicitado el enfoque de prácticas sociales de Bourdieu, es necesario aplicar su marco conceptual al fenómeno de la ayuda. En ese sentido, lo primero que se debe de hacer es explicar, a través de una narrativa histórica, el surgimiento del campo de la ayuda internacional para el desarrollo. Con tal motivo es necesario establecer algunos parámetros preliminares que puedan servir de guías para realizar la vinculación necesaria entre lo teórico y lo empírico. De esta manera, entonces, es necesario especificar, y siguiendo con lo establecido en la sección anterior, que el campo de la ayuda internacional para el desarrollo, como lugar de posiciones estructuradas, se integró por dos grupos: los países desarrollados y los países en vías de desarrollo, entre los cuales podemos 
incluir a gran parte de los donantes no tradicionales (Esteves \& Assunção, 2014, p. 1777).

En el caso de los países desarrollados, debido al capital que poseían lograron convertirse rápidamente en subordinantes, ocupando la posición más elevada en la estructura y, por ende, posicionando su visión particular de las cosas como la doxa imperante dentro del campo. Por su parte, los países en vías de desarrollo, receptores y donantes no tradicionales, constituyeron la otra cara de la moneda, el grupo que, debido a su posición, ocupó el lugar de subordinado, en momentos aceptando la doxa de los subordinantes y en otros cuestionándola, tratando de erosionar la influencia de los donantes tradicionales para avanzar su propia agenda, entrando en una batalla dóxica con los países desarrollados.

Ya que se han llevado a cabo estas aclaraciones preliminares, es necesario establecer los acontecimientos históricos que dieron forma al campo de la ayuda internacional para el desarrollo. Dicho campo se estableció tras el fin de la Segunda Guerra Mundial (Moreira, 2020), cuando los países vencedores, bajo el liderazgo de Estados Unidos (EE.UU.), se embarcaron en la titánica tarea de construir el orden económico y político de post-guerra. Dicho orden liberal hegemónico, como lo denomina Ikenberry (2011), tenía como principal propósito contener la expansión de la influencia soviética, sobre todo en Europa occidental, aunque también en América Latina, Asia y África, lo que sirvió para canalizar los intereses particulares de las principales potencias capitalistas de la época en un interés común (Gilpin, 2000/2003).

Para poder llevar a cabo dicha tarea, el Gobierno de Washington implementó el Programa de Recuperación Europeo, mejor conocido como Plan Marshall. A grandes rasgos, el Plan Marshall fue un programa de ayuda orientado a transferir recursos a las economías europeas por medio de subvenciones en dólares, subvenciones en especie y préstamos (Kunz, 1997, p. 168). Para poder administrar el dinero que llegaba desde Estados Unidos, y de forma paralela al establecimiento de la Comisión Económica para Europa dentro de las Naciones Unidas, algunos gobiernos de Europa occidental crearon, en 1948, la denominada Organización Europea de Cooperación Económica (OECE) que asemejó a un club exclusivo de países occidentales (Stinsky, 2017, p. 72).

La OECE perduró, con algunas tensiones y conflictos, hasta la década de 1960 cuando experimentó una reconfiguración, partiendo de la idea de solidificar los lazos entre Europa y Estados Unidos y mejorar la cooperación económica entre ambas partes (Wolfe, 2008, p. 27). De esta manera la OECE 
se transformó en la Organización para la Cooperación y el Desarrollo Económico (OCDE) después de que en mayo de 1960 se aprobara la admisión y plena membresía de Canadá y de Estados Unidos. La convención que dio origen a la organización fue firmada el 14 de diciembre de 1960 y entró en vigor un año más tarde, el 30 de septiembre de 1961 (Woodward, 2009, p. 18). Con esto, el Gobierno de Washington y los países de Europa occidental afianzaron su relación en el contexto de la Guerra Fría y refrendaron su compromiso de crear una verdadera alianza transatlántica, en lo político-militar con la creación de la Organización del Tratado del Atlántico Norte (OTAN) en 1949, y en el ámbito de la cooperación económica con la creación de la ya mencionada organización.

En este primer momento la organización centró sus esfuerzos en tres ámbitos específicos: comercio, gestión financiera y desarrollo (Woodward, 2009). En este último tema, y como bien señalan Leimgruber y Schmelzer (2017), la OCDE se posicionó rápidamente como uno de los principales actores a nivel internacional, situándose al mismo nivel que el Banco Internacional de Reconstrucción y Fomento (BIRF) y el Programa de Naciones Unidas para el Desarrollo (PNUD) (p. 37). Esto fue posible gracias a la creación de un subgrupo dentro de la organización que se encargó de establecer los parámetros bajo los cuales se iban a guiar los esfuerzos de los países en materia de ayuda para el desarrollo. Dicho subgrupo, heredero inmediato del denominado Grupo de Ayuda para el Desarrollo (GAD), establecido en 1960 dentro de la OECE, devino el Comité de Ayuda para el Desarrollo (CAD) (Woodward, 2009, p. 22). El CAD se encargó de "establecer la ayuda para el desarrollo como una función normal de los Estados modernos y estableció algunas de las más influyentes normas, estándares y referentes en el campo del desarrollo [...]" (Leimgruber \& Schmelzer, 2017, p. 37).

En 1972 el CAD estableció los parámetros para regular, por decirlo de alguna manera, la práctica de la ayuda a través de lo que se denominó Asistencia Oficial para el Desarrollo (AOD) que permitió distinguir, como bien menciona Furia (2015), la ayuda concesional de otros flujos (p. 71). Para que algún tipo de flujo pudiera clasificarse como AOD tenía que poseer las siguientes características: originarse de una fuente oficial, estar dirigida a un territorio o país establecido en una lista por los miembros del CAD, el financiamiento debía ser concesional en carácter, con un verdadero subsidio por parte del gobierno donante, y tener una subvención de por lo menos $25 \%$ utilizando una tasa de descuento del $10 \%$, y, además, debía de tener como principal objetivo el 
beneficio económico a países en vías de desarrollo (Bräutigam, 2011, p. 754; Furia, 2015, p. 70).

La definición establecida dentro del CAD permitió a los países desarrollados crear la idea de una "moralidad superior" por tratar de "erradicar" las desigualdades a nivel internacional (Hook \& Rumsey, 2016, p. 59), o, como menciona Hattori (2003), posicionarlos como los "contadores morales" de la ayuda (p. 241). En ese sentido, la estipulación y creación de la AOD le permitió al CAD, y por ende a los países más ricos, establecer y delimitar las ideas preponderantes dentro del campo de la ayuda. Así pues, el CAD instauró los parámetros para definir lo que sí podía considerarse ayuda y aquello que no, como el comercio, inversión o ayuda militar, demarcando el campo y estableciendo parte de la doxa del mismo (Esteves \& Assunção, 2014, p. 1777).

De esta manera, la OCDE/CAD, a través de la Asistencia Oficial para el Desarrollo, comenzó a generar una serie de pautas, normas, procedimientos y prácticas que se convirtieron, con el paso del tiempo, en el molde que adoptaría la mayoría de los participantes en el campo de la ayuda para el desarrollo. Para tener un mejor control sobre la asignación de los recursos destinados a dicha partida, se establecieron la "coordinación, armonización, monitoreo, evaluación y rendición de cuentas para programas de ayuda intencionalmente altruistas" (Dunford, 2020, p. 3) como las pautas hegemónicas que se debían de seguir. Dicho enfoque, como argumentan algunos, se organizó alrededor de una estructura compleja de consejeros y técnicos que, además de sustraer una gran cantidad del monto total de la ayuda, gozaban de ciertos privilegios para intervenir en los países receptores (Dunford, 2020, p. 3).

Algunos desarrollos puntuales, sobre todo en el ámbito económico, transformaron el panorama de la ayuda internacional, aunque dejaron casi intacta la doxa establecida previamente, añadiendo solamente algunos elementos más a la misma. La contrarrevolución conservadora, como la denomina Gilpin (2000/2003, p. 114), de la década de los ochenta marcó un nuevo hito dentro del campo, modificando, hasta cierto punto, parte de las ideas imperantes que hasta ese momento reinaban en el ámbito político y económico. El replanteamiento del papel del Estado en la economía propició un giro drástico en las prácticas de la ayuda internacional. Derivado de esto, algunos países establecieron condiciones más estrictas para la asignación de recursos. Así pues, comenzaron a condicionar la ayuda por medio de lo que se denominó programas de ajuste estructural, que consistían en una serie de requisitos que se tenían que cumplir para poder acceder a los recursos. Entre las medidas 
que dichos países tenían que realizar podemos mencionar algunas, como la estabilidad macroeconómica, liberalización del comercio y finanzas, disciplina fiscal y privatización (Dunford, 2020, p. 3; Furia, 2015, p. 52), lo que después sería conocido como el Consenso de Washington.

Como consecuencia, los donantes tradicionales y las instituciones financieras internacionales, como el Banco Mundial y el Fondo Monetario Internacional comenzaron a tener un papel cada vez mayor en la toma de decisiones de los países receptores de ayuda, sobre todo en aspectos económicos y asociados precisamente con el conjunto de ideas antes mencionadas (Jakupec \& Kelly, 2016, p. 322). A partir de la década de los noventa, a las prescripciones económicas se le se sumaron directrices políticas, denominadas condicionantes políticas, centradas en la protección y el respeto de los derechos humanos, la adopción de valores democráticos y de buena gobernanza por parte de los países receptores (Crawford, 1997, p. 69). Así pues, a través de los programas de ajuste estructural y las condicionantes políticas, los países del CAD, junto con las instituciones financieras internacionales, establecieron el marco conceptual base para definir y medir cuestiones medulares respecto del campo, como la pobreza, el desarrollo, el empoderamiento, la participación, la gobernanza, la corrupción y la calidad institucional, delimitando aún más el campo y perfeccionando la doxa vigente (Domínguez Martín, 2018, p. 88).

El fracaso de las estrategias implementadas en el campo de la ayuda orilló a los países de la OCDE/CAD a replantearse el enfoque que habían adoptado hasta ese momento. Este proceso comenzó con la reducción a los presupuestos destinados a dicho rubro durante los noventa, debido a la evidencia exponencial de que la entrega de ayuda no había generado los resultados esperados y culminó con una reunión de alto nivel realizada en la capital francesa en el año 2005, en la que se congregaron donantes tradicionales, países receptores, organizaciones de la sociedad civil, organizaciones no-gubernamentales y organismos internacionales, y se firmó la denominada Declaración de París (Brown, 2020).

Dicha declaración trató de establecer un nuevo enfoque dentro de las prácticas de ayuda, uno en el cual se reconoció una responsabilidad compartida entre países receptores y donantes (Brown, 2020). Esta nueva actitud quedó estipulada en lo que Brown (2020) denomina los "cinco de París", los principios rectores del consenso acordado en la ciudad francesa, los cuales son: propiedad, alineamiento, armonización, gestión de resultados y responsabilidad mutua. Este nuevo enfoque, denominado "nueva agenda para la 
ayuda", originó el surgimiento de la norma sobre efectividad de la ayuda que tenía como propósito matizar las ideas preponderantes hasta el momento para promover un mejor entendimiento y una mayor inclusión de todos los actores involucrados en el campo de la ayuda. A la reunión de París le siguió una realizada en Accra, Ghana, en 2008, y otra en Busan, República de Corea en el año 2011. Precisamente en Busan, las tensiones entre los diferentes actores salieron a flote y los cuestionamientos a la doxa liberal (Bony-Cisternes, 2019) se volvieron más evidentes.

\section{Los países en vías de desarrollo y los donantes no tradicionales}

Al paralelo de lo que sucedió tras la conformación de la OCDE, y el subsecuente establecimiento del CAD, una serie de actores no pertenecientes a este organismo también comenzaron a realizar tareas de entrega de ayuda alrededor del mundo. Sin embargo, y debido a la posición estructural de dicho grupo, estos "otros" participantes siempre estuvieron en constante tensión con la arquitectura imperante.

De manera general, se identifican, dentro de estos donantes no tradicionales, a tres grupos de países: 1) los países del bloque socialista, liderados por la Unión de Repúblicas Socialistas Soviéticas (URSS), que durante el contexto de la Guerra Fría se convirtieron, por lo menos ideológicamente hablando, en los principales rivales de Estados Unidos y los países de Europa occidental; 2) los países del Golfo Pérsico, que tras el embargo petrolero en los setenta vivieron una suerte de boom que lograron capitalizar en el campo de la ayuda, y, finalmente 3) los denominados donantes del Sur, grupo de países que tiene sus antecedentes en la Conferencia de Bandung y el Movimiento de los No Alineados y agrupó a la mayoría de los considerados países en vías de desarrollo (Mawdsley, 2012a). De estos tres grupos, los donantes del Sur se convirtieron, sobre todo a partir de la década de los setenta, en una voz unificada con peso en la política internacional que abogó por un cambio profundo en el campo de la ayuda.

Dicho grupo promovió la cooperación y el comercio con una perspectiva Sur-Sur y presionó para que un nuevo acuerdo económico a escala global, el nuevo orden económico internacional, pudiera adoptarse como nuevo esquema de relaciones más justas y equitativas (Mawdsley, 2012a, p. 62). En ese sentido, y como menciona Moreira (2020), los denominados donantes del Sur ocuparon un lugar relevante dentro del campo de la ayuda para el desarrollo; 
sin embargo, nunca pudieron desafiar la doxa imperante. Empero, la idea del nuevo orden económico internacional y la creación del G77 se posicionaron como los primeros intentos por cuestionar las ideas reinantes dentro del campo y, además, en señalar las desigualdades recreadas por la estructura y las prácticas dentro del mismo (Esteves \& Assunção, 2014, p. 1779), sentando las bases para subsecuentes debates, críticas, cuestionamientos y esfuerzos por reconsiderarlas.

A partir de la década de los noventa, algunos desarrollos puntuales permitieron el surgimiento de actores específicos que, con un capital creciente que les permitió situarse como jugadores importantes dentro del campo, lograron crear una serie de valores, ideas, creencias y prácticas alternativas a la doxa liberal. Los fenómenos que dieron pie a esto fueron la reducción de los presupuestos destinados a ayuda por parte de los países más ricos (Ruhil, 2015), la transformación y revaloración del concepto de "desarrollo", y el posterior surgimiento de los Objetivos de Desarrollo del Milenio (Hulme, 2009) y Objetivos de Desarrollo Sostenible pero, sobre todo, el gradual cambio en la estructura de poder a nivel internacional (Chin \& Quadir, 2012). Entre estos jugadores destacaron los miembros del denominado grupo BRICS, integrado por Brasil, Rusia, India, China y Sudáfrica, quienes lograron posicionarse como una alternativa viable al modelo de ayuda impuesta por los países de la $\mathrm{OCDE} / \mathrm{CAD}$, modificando, hasta cierto punto, la trayectoria de la gobernanza global (Schirm, 2010).

\section{Habitus: China como donante}

Del grupo BRICs, el caso de China ha destacado ampliamente debido al crecimiento en los programas de ayuda y el impacto que ha tenido el reposicionamiento del país como actor importante de la arena internacional. En ese sentido, Pekín es un ejemplo paradigmático sobre la tensión que existe dentro de la doxa liberal impuesta por el CAD y la visión de los donantes no tradicionales en torno al entendimiento del campo y las prácticas legitimadas. Para entender precisamente las prácticas de ayuda chinas y la tensión que generan, es necesario establecer entonces el otro componente de la perspectiva de prácticas bourdesiana, el habitus. Como ya hemos visto anteriormente, las prácticas se encuentran situadas en la intersección entre el campo, lo objetivo, y el habitus, lo subjetivo (Pouliot \& Mérand, 2013, p. 30). Como bien señala el autor francés, para poder clarificar las disposiciones de los agentes es ne- 
cesario trazar su trayectoria histórica dado que, como ya vimos en la primera sección, la noción de habitus se nutre de la experiencia pasada y presente de los agentes, lo que les permite establecer las prácticas presentes y futuras (Maton, 2014, p. 50).

Así pues, si queremos comprender las prácticas chinas tenemos que indagar la relación del país asiático con el campo de la ayuda internacional desde una perspectiva histórica. Para eso es necesario, antes de comenzar dicho trayecto, establecer algunas directrices para dar mayor sentido al argumento. Para el caso chino, podemos identificar tres momentos históricos clave que marcaron la presencia de dicho país en el campo de la ayuda internacional y que, por ende, tuvieron un impacto para el desarrollo de su habitus: la Conferencia de Bandung, el cisma sino-soviético, y el periodo de reformas económicas. Cada uno de estos episodios le permitió al Gobierno de Pekín desarrollar su "sentido del juego" y, como resultado, establecer sus prácticas para dicho campo.

Desde la instauración del gobierno comunista en 1949, China formó parte, de manera inmediata, del bloque comunista liderado por la URSS. Como parte de la "solidaridad de bloque", el Gobierno de Pekín implementó un programa de cooperación y ayuda en aras de fomentar dicho sentido de unidad, como menciona Copper (2016), centrando sus esfuerzos en la lucha contra las democracias occidentales. Esa primera etapa estuvo caracterizada por el otorgamiento de ayuda militar al esfuerzo bélico de Kim Il-Sung, durante la Guerra de Corea, a Albania, Laos, Cuba, Vietnam del Norte, Camboya, Mongolia y Hungría (Copper, 2016, pp. 133 y 134). En paralelo, el Gobierno chino se convirtió en receptor de ayuda, sobre todo soviética, con el afán de poder recuperarse del desastroso panorama nacional que dejó la guerra civil y, con esto, lograr modernizarse lo más rápido posible.

A la par de estos desarrollos, y para establecer su autonomía de los mandatos de Moscú, el gobierno comunista se dio a la tarea de establecer relaciones con aquellos países que no comulgaban de manera total ni con el bloque occidental ni con el bloque soviético. Con esto en mente, el Gobierno chino buscó foros que le permitieran establecer relaciones diplomáticas, políticas y económicas fuera de su "zona de influencia" natural. En ese sentido, la primer Conferencia Asiática-Africana de 1955, conocida generalmente con el nombre de Conferencia de Bandung, se convirtió en un hito dentro de la estrategia exterior china. Lo anterior se debe a que en ella el Gobierno comunista comenzó a posicionarse como uno líder dentro del denominado 
"Tercer Mundo" y, además, porque sirvió de plataforma para esbozar la visión particular de China en materia de relaciones internacionales (Acharya, 2016, p. 348). Esto quedó ejemplificado en los "cinco principios de co-existencia pacífica"5 que se convertirían, a la postre, en la piedra angular de la política exterior del país asiático y servirían como insumo para conformar su habitus y sus prácticas de ayuda.

Otro factor importante fue el rompimiento de relaciones entre Moscú y Pekín. Tras la muerte de Stalin, y derivado de una serie de desacuerdos que van desde las promesas fallidas del Gobierno ruso en torno a la cooperación militar, al apoyo que brindó Moscú a la India en su disputa fronteriza con China, pasando por una disputa ideológica y un conflicto fronterizo entre ambos países, entre otras cuestiones, el Kremlin retiró todo tipo de ayuda hacia su vecino sudoriental, lo que intensificó la búsqueda por parte del Gobierno chino de un lugar independiente en la arena internacional (Copper, 2016, p. 137). Derivado de estos acontecimientos, el entonces primer ministro chino Zhou Enlai expuso en 1964, y durante un viaje al continente africano, los "ocho principios de asistencia económica y técnica". ${ }^{6}$ En ese sentido, los ocho principios establecieron las directrices sobre la política en materia de ayuda económica exterior que guiaron, y guían hasta la fecha, la manera como Pekín aborda sus relaciones en ese rubro. A manera de resumen, el texto tenía como propósito resaltar el programa de ayuda chino, destacando el respeto

5. Los “cinco principios de co-existencia pacífica" son: 1) respeto mutuo por la soberanía y la integridad territorial; 2) no agresión; 3) no interferencia; 4) beneficio mutuo y equitativo, y 5) co-existencia pacífica.

6. Los ocho principios de asistencia económica y técnica son: 1) el Gobierno chino siempre se basa en el principio de igualdad y beneficio mutuo en la provisión de ayuda hacia otros países. Nunca ve ese tipo de ayuda de manera unilateral sino como de beneficio mutuo; 2) el Gobierno chino respeta la soberanía de los países receptores, y nunca condicionará dicha ayuda o demandará algún tipo de privilegios; 3) China provee ayuda económica en forma de préstamos con una tasa de interés cero o baja, extendiendo el tiempo límite de pago cuando sea necesario, esto con el fin de aligerar la carga del país receptor; 4) el propósito de la ayuda económica china no es hacer al país receptor dependiente, el propósito es hacer que el país receptor se embarque en el camino de la autosuficiencia y la independencia económica; 5) el Gobierno chino trata de ayudar a los países receptores en la construcción de proyectos de manera eficiente con resultados rápidos, de esta manera el país receptor podrá incrementar sus ingresos y capital acumulado; 6) el Gobierno chino provee de la mejor maquinaria y equipo de propia manufactura a precios de mercado. Si el material y el equipo no cumplen con los estándares adecuados, el Gobierno chino se compromete a reemplazarlos; 7) cuando se provea de asistencia técnica, el Gobierno chino se asegurará de que el personal del país receptor domine dicha técnica, y 8) los expertos enviados por China para ayudar a la construcción en los países receptores tendrán el mismo nivel de vida de los trabajadores locales. Los expertos chinos no podrán hacer ningún tipo de petición especial ni gozarán de algún privilegio. 
de la soberanía, el beneficio mutuo y el propósito de hacer autosuficientes a los países receptores (Bräutigam, 2008, p. 8; Ubi, 2014, p. 245).

A partir de aquí, la ayuda que brindó el Gobierno comunista a otros países creció de manera considerable. Para finales de 1960 y principios de 1970, el Gobierno chino tenía contemplados 20 países para construir 313 proyectos completos (Li \& Wu, 2011, p. 30). Es importante recalcar que aun cuando la economía china se encontraba en condiciones poco favorables para mantener una política de ayuda hacia el exterior, el Gobierno comunista, bajo el liderazgo de Mao Zedong, favoreció dicha estrategia porque podía mejorar la imagen del país y, además, servir para contrarrestar la influencia tanto de Estados Unidos como de la Unión Soviética. De igual manera, y después de ser admitido en la Organización de las Naciones Unidas, Pekín utilizó la ayuda internacional para aislar a Taipei, que se encontraba bajo control del Kuomintang desde el fin de la guerra civil china, y, de esta manera, coartar su política de reconocimiento.

A partir de la década de los ochenta, y con la llegada de Deng Xiaoping y los reformadores, el país experimentó un cambio radical que marcó la posterior trayectoria del gigante asiático. Tras el arribo de los "nuevos mandarines", el país asiático enfocó todos sus esfuerzos a la revigorización de la economía interna. Bajo el lema de las "cuatro modernizaciones", la élite política buscó impulsar la agricultura, la industria, ciencia y tecnología, y la defensa nacional, dejando de lado el ímpetu internacionalista que se había mostrado hasta ese momento. El reajuste político chino no significó el final de la ayuda hacia el exterior, aunque sí un decrecimiento. La nueva élite política china buscó dar una nueva dimensión a la forma como se otorgaba ayuda económica, tomando en consideración las nuevas necesidades del gigante asiático. Cabe mencionar que, durante dicha transición, China se relacionó de manera estrecha con PNUD y, además, con el Banco Mundial, aceptando ayuda tanto de Japón como de Alemania y convirtiéndose en uno de los países más grandes en materia de recepción de ayuda internacional (Zhou, 2017, p. 18).

La adopción de las reformas orientadas al mercado, aunadas el gran esfuerzo de las autoridades por transformar al país, permitieron que China pronto se convirtiera en una locomotora económica difícil de contener. Esto se logró siguiendo un enfoque de prueba y error, poniendo énfasis en el desarrollo de una base productiva como palanca para el desarrollo económico y social. Además, y derivado del cambio en el enfoque de la política exterior china, del internacionalismo desmedido de Mao a la actitud más cautelosa 
de Deng, la cúpula política adoptó una visión pragmática de las relaciones internacionales, buscando conciliar las expectativas externas con los intereses internos, la cual impera hasta el día de hoy.

Así pues, como hemos podido observar, la experiencia vital china estuvo marcada por su ímpetu por establecer su autonomía vis a vis la Unión Soviética, ser uno de los líderes del denominado "Tercer Mundo", ahora conocido como "Sur global", y el inaudito crecimiento económico que ha estado experimentando desde principios de los noventa. Derivado de estos acontecimientos, el Gobierno ha forjado una estrategia de política exterior que constituye la materialización de los desarrollos históricos que experimentó el país asiático y de su particular "sentido del juego". Esto queda ejemplificado en los cinco principios de coexistencia pacífica y los ocho principios para la ayuda económica y la asistencia técnica. En ese sentido, todo lo anterior constituye el habitus chino.

\section{China en África}

Como se ha hecho mención anteriormente, el caso africano en torno a las prácticas de ayuda chinas es sumamente importante. Esto deriva de la relevancia que ha obtenido el continente para el gigante asiático en los últimos años. Esto queda ejemplificado, por ejemplo, si tomamos en consideración que China se ha convertido, de manera individual, en el mayor donante al continente africano, por encima de Estados Unidos (Broich et al., 2020, p. 34; Tjønneland, 2020) y que para 2018, dentro del mecanismo conocido como el Foro de Cooperación China-África (FOCAC, por sus siglas en inglés), el Gobierno de Pekín se comprometió a entregar 60 mil millones de dólares americanos para el continente (Isaksson \& Kotsadam, 2020), distribuidos de la siguiente manera: 20 mil millones en nuevas líneas de crédito, 15 mil millones en ayuda exterior bajo los estándares de la OCDE/CAD, ${ }^{7} 10$ mil millones en un

7. Cabe mencionar que, como bien apunta Carter (2017), la tarea de recopilar información fidedigna en torno a los montos de ayuda que otorga el Gobierno chino es difícil de lograr. Lo anterior se debe, en gran medida, a las marcadas diferencias entre las normas que rigen a los países del cAD y las normas que rigen al país asiático y, además, a la opacidad de Pekín en torno a dicho tema. En ese sentido, es importante recalcar que para evitar confusiones y estimaciones erróneas, se ha decidido omitir hacer una comparación entre los montos de ayuda que otorga el CAD y el Gobierno chino y centrar la atención, siguiendo el objetivo del trabajo, en la manera como se proporciona la ayuda. Sin embargo, si el lector se encuentra interesado en realizar dicha labor, existen recursos que pueden ser de su interés, como AidData y el China-Africa Research Institute de la Universidad John Hopkins. 
fondo especial para financiar el desarrollo y, finalmente, cinco mil millones para un fondo especial para financiar importaciones africanas, mientras que los 10 mil millones restantes se canalizarán a través de las empresas chinas (Benabdallah \& Robertson, 2018; Tjønneland, 2020).

Con esto en consideración lo primero que hay que establecer son el tipo de prácticas que China lleva a cabo para el caso africano. En ese sentido hay que destacar, de manera anticipada, que China muestra un enfoque distinto al de los donantes tradicionales, tomando como molde su propia experiencia de desarrollo, ejemplificada en las reformas económicas impulsadas por el propio Estado que se posicionan como una vía media entre la economía liberal abierta y una planificada (Deyassa, 2019, p. 397). Así pues, el Gobierno chino para el caso africano enfatiza la asignación de recursos que contribuyan al aparato productivo de los países, aquéllos denominados como tangibles económicos, centrando sus esfuerzos en la productividad de la agricultura, los procesos industriales, el reacondicionamiento de infraestructura industrial y la construcción de hospitales y escuelas (Broich et al., 2020, p. 44). También ha proveído recursos para obras de carácter más social y deportivo, como complejos deportivos, con la denominada "diplomacia de estadio" (Xue et al., 2019). En ese sentido, y como bien señala du Plessis (2016), para el caso específico de África, China ha priorizado la construcción de infraestructura para poder zanjar el problema del desarrollo.

Otro punto importante, y en consonancia con lo anterior, es que para el caso africano la entrega de ayuda china se encuentra fuertemente ligada al comercio y la inversión, lo cual contrasta de manera explícita con la concepción de Asistencia Oficial para el Desarrollo establecida por la OCDE/ CAD. Dicha integración responde a la perspectiva china de que el desarrollo social y el desarrollo económico se encuentran fuertemente relacionados y, además, de que el desarrollo social puede ser alcanzado únicamente mediante el desarrollo económico. Así pues, la racionalidad que exhibe Pekín en torno a sus prácticas de ayuda en África postula que la reducción de la pobreza y el desarrollo social se encuentran asegurados por medio de las actividades productivas que contribuyen al desarrollo económico y a la oportunidad de empleo (Deyassa, 2019, p. 406).

Además de esto, cabe mencionar que la ayuda china no muestra condicionalidad tan férrea hacia sus pares africanos. En ese sentido, la ayuda china muestra mayor flexibilidad en torno a las condiciones de los países africanos receptores, mostrando poco interés por la situación de los derechos humanos, 
la gobernanza, los valores democráticos, etc. De igual manera, y en consonancia con su propio modelo de desarrollo económico, el Gobierno no impone ningún tipo de "fórmula" específica para que los países puedan alcanzar su desarrollo. Empero, esto no significa que no existan requisitos para la entrega de ayuda. Pekín, a diferencia de los donantes tradicionales, no impone condiciones políticas, pero sí condicionantes económicas (Chan \& Lee, 2017). Así pues, los países receptores de ayuda china deben de utilizar empresas chinas para obras de infraestructura, comprar insumos chinos, emplear mano de obra china, además de dar acceso a Pekín a recursos naturales y materias primas que se encuentran dentro de sus territorios (Broich et al., 2020, p. 44).

Lo anterior queda constatado, por ejemplo, si observamos los principales vínculos que tiene Pekín en el continente africano. De esa manera, los países que cuentan con gran cantidad de recursos naturales, entre los que destacan Angola, Sudán, Nigeria y la República Democrática del Congo, muestran una relación estrecha con los programas de ayuda chino. Dicha actitud ha sido señalada por solapar regímenes autocráticos, debido a que su política de no injerencia en los asuntos domésticos le permite movilizar recursos a Estados que bajo la lupa de los estándares de la OCDE/CAD no pueden ser candidatos a recibir este tipo de ayuda (Broich et al., 2020, p. 39). Debido a las características de algunos países, el Gobierno chino ha sido acusado de apoyar regímenes parias, minando los esfuerzos en conjunto que la comunidad internacional ha estado realizando para mejorar las prácticas democráticas de algunos gobiernos africanos y entorpeciendo los avances en materia de protección a los derechos humanos (Woods, 2008). Sin embargo, existen algunos indicios de que, por el contrario, enfatizan que la entrada de capital chino ha propiciado la mejora de las tasas de crecimiento, mejores términos de comercio, incremento en el monto de sus exportaciones y mejores ingresos públicos (Goldstein et al., 2006; Reisen, 2007; Woods, 2008, p. 1208).

Todo esto ha sido cimentado bajo un discurso peculiar, que posiciona a la ayuda china en una trayectoria completamente diferente a la de los donantes tradicionales. Así pues, a partir de su autoconcepción como el país en vías de desarrollo más grande del mundo, el Gobierno de Pekín plantea que sus programas son de cooperación para el desarrollo, en los cuales se incluye la ayuda externa, aunque también la cooperación para el desarrollo económico, que incluye las áreas de comercio e inversión, enmarcando las prácticas 
chinas como parte de la cooperación Sur-Sur, ${ }^{8}$ y que emana del denominado "espíritu de Bandung"' (Vadell et al., 2020). Esta idea discursiva genera que la relación con los países africanos se aleje de la concepción tradicional de la cooperación Norte-Sur, la cual, de acuerdo con Mawdsley (2012b), se basa en la noción maussiana del "regalo", que establece una dominación simbólica entre los donantes y los receptores de dicha ayuda.

\section{Conclusiones}

Como hemos podido observar, las prácticas chinas en el campo de la ayuda para el desarrollo representan un serio desafío para las pautas establecidas por la OCDE/CAD. Como bien señala Zhang (2020), las prácticas de ayuda que ha establecido Pekín en África, y en otras partes del mundo, confrontan de manera directa la arquitectura vigente, impuesta después de la Segunda Guerra Mundial y centrada en los países desarrollados, debido a que alienta a los países receptores a buscar fuentes alternativas de financiamiento que reduzcan sustancialmente los compromisos que deben adquirir dichos países, sobre todo en materia de gobernanza y derechos humanos, con los donantes, erosionando la influencia, hasta cierto punto, de los donantes tradicionales (p. 176).

En ese sentido, podemos argumentar que el enfoque de prácticas derivado de Bourdieu brinda herramientas valiosas para comprender la génesis de dichas acciones sociales. Como ya se ha señalado, tanto el campo de la ayuda para el desarrollo como el propio habitus del país asiático han sido un factor fundamental para entender dichas prácticas. Así pues, el surgimiento del campo de la ayuda, estructurado en dos posiciones, subordinantes, países pertenecientes a la OCDE/CAD, y subordinados, todos los demás Estados del sistema internacional, propició la legitimación de una serie de prácticas y el establecimiento de lo que se ha denominado doxa liberal. En contraposición y a la par de estos desarrollos, los países subordinados, dentro de los cuales

8. La cooperación Sur-Sur hace referencia a un modelo de cooperación que enfatiza la transferencia e intercambio de recursos, tecnología y conocimiento, construida alrededor de una serie de similitudes históricas, como su pasado colonial y su experiencia post-colonial, e identidades compartidas, basada en una idea de solidaridad y desarrollo común por parte de los países que conforman el Sur global (Mawdsley, 2019).

9. Se conoce como "espíritu de Bandung" a la idea de solidaridad que se gestó después de la conferencia Asiático-Africana de 1955 y tenía la idea de cambiar las "reglas de juego" a nivel internacional, proponiendo modelos de cooperación político-económica alternativos a los impuestos por los países industrializados (Weber \& Winanti, 2016). 
China era una pieza importante, comenzaron a generar prácticas alternas y visiones fuera del marco hegemónico. Sin embargo, y debido al capital -económico, social, cultural y simbólico- de dicho grupo, éstos no lograron, en una primera instancia, tener mucha resonancia para generar un cambio en la doxa imperante.

Mientras esto sucedía, la República Popular de China experimentó una serie de acontecimientos que marcarían su experiencia vital, o habitus, y, por ende, su entendimiento del campo. Respecto a esto, podemos mencionar como puntos importantes para entender su trayectoria: su integración al bloque independiente en el contexto de la Guerra Fría; con su asistencia a la Conferencia de Bandung y la enunciación del programa de los cinco principios de co-existencia pacífica; así como su rompimiento con el bloque socialista y su establecimiento como donante, que queda ejemplificado en los ocho principios para la ayuda económica y la asistencia técnica. Esto se encuentra enmarcado en la integración de China con el denominado "Sur global", que a través de iniciativas como el nuevo orden económico internacional y el plan de acción para promover e implementar la cooperación técnica entre los países en vías de desarrollo, buscaron, como se mencionó anteriormente, soluciones adecuadas al problema del subdesarrollo y la desigualdad a nivel global.

Todo esto ha sido complementado con el crecimiento económico que ha experimentado el país asiático. Gracias a su propia trayectoria de desarrollo económico, China ha incorporado dicha visión, sin un esquema predefinido y aludiendo a la prueba y error, dentro de su habitus, lo cual se ve reflejado en sus prácticas. Además, el crecimiento exponencial de su capital económico, simbólico y social derivado de este hecho le ha permitido cuestionar de manera preocupante el andamiaje institucional preponderante, disputando la doxa establecida.

\section{Referencias bibliográficas}

Acharya, A. (2016). Studying the Bandung conference from a Global IR perspective. Australian Journal of International Affairs, 70(4), 342-357. https:// doi.org/10.1080/10357718.2016.1168359

Adler, E., \& Pouliot, V. (2011). International practices. International Theory, 3(1), 1-36. https://doi.org/10.1017/S175297191000031X 
Adler-Nissen, R. (2011). On a Field Trip with Bourdieu. International Political Sociology, 5(3), 327-330. https://doi.org/10.1111/j.17495687.2011.00137_1.x

Adler-Nissen, R. (2012). Introduction. En R. Adler-Nissen (Ed.), Bourdieu in International Relations: Rethinking Key Concepts in IR (pp.1-23). Routledge. https://doi.org/10.4324/9780203102282

Aedo Henríquez, A. (2014). El habitus y la movilidad social: de la modificación del sistema de disposiciones a la transformación de la estructura de clases. Revista de Sociología, (29), 57-75. https://doi.org/10.5354/0719529X.2014.36178

Beeson, M. (2013). The rise of China and the future of international political economy. En R. Palan (Ed.), Global political economy (2a ed., pp. 232-243). Routledge. https://doi.org/10.4324/9780203097762

Benabdallah, L., \& Robertson W. (2018, septiembre 17). Xi Jinping pledged $\$ 60$ billion for Africa. Where will the money go? The Washington Post. https://www.washingtonpost.com/news/monkey-cage/wp/2018/09/17/ xi-jinping-pledged-60-billion-for-africa-where-will-the-money-go/

Bigo, D. (2013). Pierre Bourdieu y las Relaciones Internacionales: el poder de las prácticas, las prácticas del poder. Relaciones Internacionales, (24), 3376. https://revistas.uam.es/relacionesinternacionales/article/view/5191 Bony-Cisternes, R. (2019). Les mutations de l'aide publique au développement au Vietnam au travers du prisme de la gouvernance [Changes in official development assistance in Vietnam as seen through the prism of governance]. International Development Policy | Revue Internationale de Politique de Développement 11.1. https://doi.org/10.4000/poldev.3204

Bourdieu, P. (1997). Razones prácticas. Sobre la teoría de la acción (Trad. Thomas Kauf). Anagrama. (Trabajo original publicado en 1994).

Bourdieu, P. (2000). Cosas dichas (Trad. Margarita Mizraji). Gedisa. (Trabajo original publicado en 1987).

Bourdieu, P., \& Wacquant, L. (2008). Una invitación a la sociología reflexiva (Trad. Ariel Dilon). Siglo veintinuno editores. (Trabajo original publicado en 1992).

Bräutigam, D. (2008). China's African Aid: transatlantic challenges. German Marshall Fund of the United States.

Bräutigam, D. (2011). Aid 'With Chinese Characteristics': Chinese Foreign Aid and Development Finance Meet the OECD-DAC Aid Regime. Journal 
of International Development, 23(5), 752-764. https://doi.org/10.1002/ jid.1798

Broich, T., Szirmai, A., \& Adedokun, A. (2020). Chinese and Western Development Approaches in Africa: Implications for the SDGs. En M. Ramutsindela \& D. Mickler (Eds), Africa and the Sustainable Development Goals (pp.33-48). Springer International Publishing. https://doi. org/10.1007/978-3-030-14857-7

Brown, S. (2020). The Rise and Fall of the Aid Effectiveness Norm. The European Journal of Development Research, (32), 1230-1248. https://doi. org/10.1057/s41287-020-00272-1

Bueger, C., \& Gadinger, F. (2015). The Play of International Practice. International Studies Quarterly, 59(3), 449-460. https://doi.org/10.1111/isqu.12202

Carter, B. (2017, september). A literature review of China's Aid (K4D Helpdesk Report). Institute of Development Studies. https://gsdrc.org/ publications/a-review-of-chinas-official-development-assistance/

Cerón-Martínez, A. U. (2019). Habitus, campo y capital. Lecciones teóricas y metodológicas de un sociólogo bearnés. Cinta de Moebio. Revista de Epistemología de Ciencias Sociales, (66), 310-320. https://cintademoebio. uchile.cl/index.php/CDM/article/view/55912

Chan, L.H., \& Lee, P. K. (2017). Power, Ideas and Institutions: China's Emergent Footprints in Global Governance of Development Aid (CSGR Working Paper 281/17). The University of Warwick. https://warwick.ac.uk/fac/soc/pais/ research/researchcentres/csgr/papers/281-17.pdf

Chihu-Amparán, A. (1996). La teoría de los campos en Pierre Bourdieu. Polis, (98), 179-200. https://polismexico.izt.uam.mx/index.php/rp/article/ view/345

Chin, G., \& Quadir, F. (2012). Introduction: rising states, rising donors and the global aid regime. Cambridge Review of International Affairs, 25(4), 493-506. https://doi.org/10.1080/09557571.2012.744642

Copper, J. (2016). China's Foreign Aid and Investment Diplomacy (Vol. I). Palgrave Macmillan. https://doi.org/10.1057/9781137532732

Cornut, J. (2017, November 20). The Practice Turn in International Relations Theory. En Oxford Research Encyclopedia of International Studies. International Studies Association and Oxford University Press. https://doi. org/10.1093/acrefore/9780190846626.013.113 
Crawford, G. (1997). Foreign aid and political conditionality: Issues of effectiveness and consistency. Democratization, 4(3), 69-108. https://doi. org $/ 10.1080 / 13510349708403526$

Deyassa, K. G. (2019). To what extent does China's aid in Africa affect traditional donors? International Journal of Sociology and Social Policy, 39(5/6), 395-411. https://doi.org/10.1108/IJSSP-01-2019-0003

Domínguez-Martín, R. (2018). Dialéctica del origen, despliegue y climaterio del régimen internacional de la ayuda. Revista de Economía Crítica, 25, 76-104. http://www.revistaeconomiacritica.org/sites/default/files/7RafelDominguez_Regimen-internacional-de-ayuda.pdf

du Plessis, R. (2016, October 3). China's African Infrastructure Projects: A Tool in Reshaping Global Norms (SAIIA Policy Insights No 35). South African Institute of International Affairs. https://saiia.org.za/research/chinasafrican-infrastructure-projects-a-tool-in-reshaping-global-norms/

Dunford, M. (2020). Chinese and Development Assistance Committee (DAC) development cooperation and development finance: implications for the BRI and international governance. Eurasian Geography and Economics, 61(2), 125-136. https://doi.org/10.1080/15387216.2020.1716821

Esteves, P., \& Assunção, M. (2014). South-South cooperation and the international development battlefield: between the OECD and the UN. Third World Quarterly, 35(10), 1775-1790. https://doi.org/10.1080/0143659 7.2014.971591

Furia A. (2015). The Foreign Aid Regime: Gift-Giving, States and Global Dis/ Order. Palgrave Pivot. https://doi.org/10.1057/9781137505903

Gilpin, R. (2003). El reto del capitalismo global. La economía mundial en el siglo XXI (Trad. Pablo Gianera). Oceano. (Trabajo original publicado en 2000). Givens, J. W. (2011). The Beijing Consensus is Neither: China as a NonIdeological Challenge to International Norms. St Antony's International Review, 6(2), 10-26. http://www.jstor.org/stable/26226762

Goldstein, A., Pinaud, N., Reisen, H., \& Chen, X. (2006). The rise of China and India: what's In It for Africa? OECD. https://www.oecd.org/dev/theriseofchinaandindia-whatsinitforafrica.htm

Hattori, T. (2003). The moral politics of foreign aid. Review of International Studies, 29(2), 229-247. https://doi.org/10.1017/S0260210503002298 Hook, S. W., \& Rumsey, J. G. (2016). The Development Aid Regime at Fifty: Policy Challenges Inside and Out. International Studies Perspectives, 17(1), 55-74. https://doi.org/10.1111/insp.12101 
Hulme, D. (2009). The Millennium Development Goals (MDGs): A Short History of the World's Biggest Promise (BWPI Working Paper No. 100). Social Science Research Network. https://ssrn.com/abstract $=1544271$

Ikenberry, G. (2011). Liberal Leviathan: The Origins, Crisis, and Transformation of the American World Order. Princeton University Press.

Isaksson, A.-S., \& Kotsadam, A. (2020). Chinese Aid to Africa: Distinguishing Features and Local Effects (IFN Working Paper No. 1337, 2000). Social Science Research Network. https://ssrn.com/abstract=3643781

Jakupec V., \& Kelly, M. (2016) Development Aid: Regulatory Impact Assessment and Conditionality. Impact Assessment and Project Appraisal, 34(4), 319-329. https://doi.org/10.1080/14615517.2016.1228339

Kratochwil, F. (2011). Making sense of "international practices". En E. Adler \& V. Pouliot (Eds.), International practices (pp.36-60). Cambridge University Press. https://doi.org/10.1017/CBO9780511862373.004

Kunz, D. B. (1997). The Marshall Plan Reconsidered: A Complex of Motives. Foreign Affairs, 76(3), 162-170.

Leimgruber, M., \& Schmelzer, M. (2017). From the Marshall Plan to Global Governance: Historical Transformations of the OEEC/OECD, 1948 to Present. En M. Leimgruber \& M. Schmelzer (Eds.), The OECD and the International Political Economy Since 1948 (pp. 23-61). Palgrave Macmillan. https://doi.org/10.1007/978-3-319-60243-1

Li, X., \& Wu, J. (2011). China's foreign aid: Historical evolution, architecture and challenges. En Korea Development Institute / The Asia Foundation (Eds.), Emerging Asian approaches to development cooperation, (pp.27-37). Korea Development Institute; The Asia Foundation. https://www.kdi. re.kr/kdi_eng/publications/publication_view.jsp?pub_no=12158

Loyal, S., \& Quilley, S. (2017). The particularity of the universal: critical reflections on Bourdieu's theory of symbolic power and the state. Theory and Society, 46, 429-462. https://doi.org/10.1007/s11186-017-9298-y

Maton, K. (2014). Habitus. En M. Grenfell, (Ed.). Pierre Bourdieu: Key Concepts (2a ed.) (pp.48-64). Routledge. https://doi.org/10.4324/9781315729923 Mawdsley, E. (2012a) From Recipients to Donors: Emerging Powers and the Changing Development Landscape. Zed Books https://doi.org/10.1111/ ecge.12035

Mawdsley, E. (2012b). The changing geographies of foreign aid and development cooperation: contributions from gift theory. Transactions of the 
Institute of British Geographers, 37(2), 256-272. https://doi.org/10.1111/ j.1475-5661.2011.00467.x

Mawdsley, E. (2019). South-South Cooperation 3.0? Managing the consequences of success in the decade ahead. Oxford Development Studies, 47(3), 259-274. https://doi.org/10.1080/13600818.2019.1585792

Moreira, A. (2020). From the paradigmatic to the practical battlefield: Southern development cooperation practices in a traditional aid hosting context. Revista Brasileira de Política Internacional, 63(2), https://doi. org/10.1590/0034-7329202000204

Neumann, I. B. (2002). Returning Practice to the Linguistic Turn: The Case of Diplomacy. Millennium: Journal of International Studies, 31(3), 627-651. https://doi.org/10.1177/03058298020310031201

Pouliot, V. (2012). Methodology. En R. Adler-Nissen (Ed.), Bourdieu in international relations: rethinking key concepts in IR (pp.1-14). Routledge. https:// doi.org/10.4324/9780203102282

Pouliot, V., \& Mérand, F. (2012). Bourdieu's concepts. En R. Adler-Nissen (Ed.), Bourdieu in international relations: rethinking key concepts in IR (pp.1-23). Routledge. https://doi.org/10.4324/9780203102282

Reisen, H. (2007). Is China Actually Helping Improve Debt Sustainability in Africa? (G-24 Policy Brief No. 9). Intergovernmental Group of Twenty Four. https://www.g24.org/wp-content/uploads/2013/03/G24-PolicyBrief-9.pdf

Ruhil, R. (2015). Millennium Development Goals to Sustainable Development Goals: Challenges in the Health Sector. International Studies, 52(1-4), 118-135. https://doi.org/10.1177/0020881717725926

Schirm, S. A. (2010). Leaders in need of followers: Emerging powers in global governance. European Journal of International Relations, 16(2), 197-221. https://doi.org/10.1177/1354066109342922

Stinsky, D. (2017). Western European Vs. All-European Cooperation? The OEEC, the European Recovery Program, and the United Nations Economic Commission for Europe (ECE), 1947-1952. En M. Leimgruber \& M. Schmelzer (Eds.), The OECD and the International Political Economy Since 1948, (pp. 65-88). Palgrave Macmillan. https://doi.org/10.1007/978-3319-60243-1

Tjønneland, E. (2020). The changing role of Chinese development aid. CMI Insight, (2), 1-8. https://www.cmi.no/publications/7173-the-changingrole-of-chinese-development-aid 
Ubi, E. N. (2014). Foreign Aid and Development in Sino-African Relations. Journal of Developing Societies, 30(3), 243-272. https://doi. org/10.1177/0169796X14536971

Vadell, J., Brutto, G. L., \& Leite, A. C. C. (2020). The Chinese South-South development cooperation: an assessment of its structural transformation. Revista Brasileira de Política Internacional, 63(2). https://doi. org/10.1590/0034-7329202000201

Weber, H. \& Winanti, P. (2016). The 'Bandung spirit' and solidarist internationalism. Australian Journal of International Affairs, 70(4), 391-406. https://doi.org/10.1080/10357718.2016.1167834

Wolfe, R. (2008). From Reconstructing Europe to Constructing Globalization: The OECD in Historical Perspective. En R. Mahon \& S. McBride (Eds.), The OECD and Transnational Governance, (pp. 25-42). UBC Press.

Woods, N. (2008). Whose aid? Whose influence? China, emerging donors and the silent revolution in development assistance. International Affairs, 84(6), 1205-1221. http://www.jstor.org/stable/25144989

Woodward, R. (2009). The Organisation for Economic Co-operation and Development (OECD). Routledge. https://doi.org/10.4324/9780203875773

Xue, C. Q. L., Ding, G., Chang, W., \& Wan, Y. (2019). Architecture of "Stadium Diplomacy" - China-aid sport buildings in Africa. Habitat International, 90. https://doi.org/10.1016/j.habitatint.2019.05.004

Zhang, D. (2020). Chinese Foreign Aid and Financing: An Example of New Development Assistance? En Y. Jing, A. Mendez \& Y. Zheng (Eds.), New Development Assistance: Emerging Economies and the New Landscape of Development Assistance (pp. 167-180). Palgrave Macmillan. https://doi. org/10.1007/978-981-13-7232-2

Zhou, H. (2017). China's Foreign Aid Policy and Mechanisms. En H. Zhou \& H. Xiong (Eds.), China's Foreign Aid: 60 Years in Retrospect (pp.1-47). Springer. https://doi.org/10.1007/978-981-10-2128-2 